Pacific

Journal of

Mathematics

A GENERALIZATION OF THE GREENE-KRANTZ THEOREM FOR THE SEMICONTINUITY PROPERTY OF AUTOMORPHISM GROUPS

\author{
JAE-CHEON JOO
}




\title{
A GENERALIZATION OF THE GREENE-KRANTZ THEOREM FOR THE SEMICONTINUITY PROPERTY OF AUTOMORPHISM GROUPS
}

\author{
JAE-CHEON JOO
}

\begin{abstract}
We give a CR version of the Greene-Krantz theorem (Math. Ann. 261:4 (1982), 425-446) for the semicontinuity of complex automorphism groups. This is not only a generalization but also an intrinsic interpretation of the GreeneKrantz theorem.
\end{abstract}

\section{Introduction}

By upper semicontinuity, or simply semicontinuity, in geometry, we mean the property that the set of symmetries of a geometric structure should not decrease at a limit of a sequence of the structures. For instance, a sequence of ellipses in the Euclidean plane can converge to a circle, while a sequence of circles cannot converge to a noncircular ellipse. This property seems as natural as the second law of thermodynamics in physics, but we still need to make it clear in mathematical terminology. A symmetry for a geometric structure is described as a transformation on a space with the geometric structure. The set of transformations becomes a group with respect to the composition operator. Therefore, semicontinuity can be understood as a nondecreasing property of the transformation group at the limit of a sequence of geometric structures. One of the strongest descriptions of semicontinuity was obtained by Ebin for the Riemannian structures on compact manifolds in terms of conjugations by diffeomorphisms.

Theorem 1.1 [Ebin 1970]. Let $M$ be a $C^{\infty}$-smooth compact manifold and let $\left\{g_{j}: j=1,2, \ldots\right\}$ be a sequence of $C^{\infty}$-smooth Riemannian structures which converges to a Riemannian metric $g_{0}$ in the $C^{\infty}$ sense. Then for each sufficiently large $j$, there exists a diffeomorphism $\phi_{j}: M \rightarrow M$ such that $\phi_{j} \circ I_{j} \circ \phi_{j}^{-1}$ is a Lie subgroup of $I_{0}$, where $I_{j}$ and $I_{0}$ represent the isometry groups for $g_{j}$ and $g_{0}$, respectively.

The group of holomorphic automorphisms on a complex manifold plays the role of the group of symmetries with respect to the complex structure. By Cartan's

MSC2010: 32M05, 32V05, 53C56.

Keywords: semicontinuity property, CR structures, CR Yamabe equation, automorphism groups. 
theorem (cf. [Greene et al. 2011]), the automorphism group of a bounded domain in the complex Euclidean space turns out to be a Lie group with the compact-open topology on the domain. Greene and Krantz proved the following theorem for the semicontinuity property of automorphism groups of bounded strongly pseudoconvex domains.

Theorem 1.2 [Greene and Krantz 1982]. Let $\Omega_{j}(j=1,2, \ldots)$ and $\Omega_{0}$ be bounded strongly pseudoconvex domains in $\mathbb{C}^{n}$ with $C^{\infty}$-smooth boundary. Suppose that $\Omega_{j}$ converges to $\Omega_{0}$ in the $C^{\infty}$ sense, that is, there exists a diffeomorphism $\psi_{j}$ defined on a neighborhood of $\bar{\Omega}_{0}$ into $\mathbb{C}^{n}$ such that $\psi_{j}\left(\Omega_{0}\right)=\Omega_{j}$ and $\psi_{j} \rightarrow$ Id in the $C^{\infty}$ sense on $\bar{\Omega}_{0}$. Then for every sufficiently large $j$, there exists a diffeomorphism $\phi_{j}: \Omega_{j} \rightarrow \Omega_{0}$ such that $\phi_{j} \circ \operatorname{Aut}\left(\Omega_{j}\right) \circ \phi_{j}^{-1}$ is a Lie subgroup of $\operatorname{Aut}\left(\Omega_{0}\right)$.

Unlike the isometry group of a compact Riemannian manifold, the holomorphic automorphism group on a bounded strongly pseudoconvex domain can be noncompact, so the proof of Theorem 1.2 is divided into two cases: either $\operatorname{Aut}\left(\Omega_{0}\right)$ is compact or it is not. It turns out that the latter case is relatively simple, which is the case of deformations of the unit ball by the Wong-Rosay theorem [Rosay 1979; Wong 1977]. The main part of the proof of Theorem 1.2 is thus devoted to the case when $\operatorname{Aut}\left(\Omega_{0}\right)$ is compact. Greene and Krantz proved this case by constructing a compact Riemannian manifold $\left(M, g_{j}\right)$ which includes $\Omega_{j}$ as a relatively compact subset and whose isometry group contains the automorphism group of $\Omega_{j}$. Then Ebin's theorem yields the conclusion. The Riemannian manifold $\left(M, g_{j}\right)$ is called a metric double of $\Omega_{j}$.

The idea of this proof is applicable to more general cases. One reasonable generalization is to prove the semicontinuity property for a more general class of domains. In a recent paper [Greene et al. 2013], the authors generalized Theorem 1.2 to finitely differentiable cases. Greene and Kim [2014] proved that a partial generalization is also possible even for some classes of nonstrongly pseudoconvex domains. See also [Krantz 2010] for this line of generalization.

The aim of the present paper is to obtain another generalization of Theorem 1.2. According to Hamilton's theorem [1977; 1979], deformations of a bounded strongly pseudoconvex domain with $C^{\infty}$-smooth boundary coincide with deformations of a complex structure on a given domain and they give rise to deformations of the CR structure of the boundary. Fefferman's extension theorem [1974] shows that every holomorphic automorphism on a bounded strongly pseudoconvex domain with $C^{\infty}$ smooth boundary extends to a diffeomorphism up to the boundary and hence gives rise to a CR automorphism on the boundary. Conversely, a CR automorphism on the boundary extends to a holomorphic automorphism on the domain by the BochnerHartogs extension theorem. It is also known that the compact-open topology of the automorphism group of the domain coincides with the $C^{\infty}$-topology of the 
CR automorphism group of the boundary (cf. [Bell 1987]) if the holomorphic automorphism group of the domain is compact. In this observation, it is natural to think of the semicontinuity property for abstract strongly pseudoconvex CR manifolds under deformations of CR structures as a generalization of Theorem 1.2. We prove the following theorem for CR automorphism groups when the limit structure has a compact $\mathrm{CR}$ automorphism group.

Theorem 1.3. Let $\left\{J_{k}: k=1,2, \ldots\right\}$ be a sequence of $C^{\infty}$-smooth strongly pseudoconvex $C R$ structures on a compact differentiable manifold $M$ of dimension $2 n+1$ which converges to a $C^{\infty}$-smooth strongly pseudoconvex $C R$ structure $J_{0}$ on $M$ in the $C^{\infty}$ sense. Suppose that the CR automorphism group $\operatorname{Aut}_{\mathrm{CR}}\left(M, J_{0}\right)$ is compact. Then there exists $N>0$ and a diffeomorphism $\phi_{k}: M \rightarrow M$ for each $k>N$ such that $\phi_{k} \circ \operatorname{Aut}_{\mathrm{CR}}\left(M, J_{k}\right) \circ \phi_{k}^{-1}$ is a Lie subgroup of $\operatorname{Aut}_{\mathrm{CR}}\left(M, J_{0}\right)$.

According to Schoen's theorem [1995], $\operatorname{Aut}_{\mathrm{CR}}\left(M, J_{0}\right)$ is compact if and only if $\left(M, J_{0}\right)$ is not CR equivalent to the sphere $S^{2 n+1}$ with the standard CR structure. One should notice that this condition is not necessary if $2 n+1 \geq 5$. Boutet de Monvel [1975] showed that a CR structure on $M$ which is sufficiently close to the standard structure on $S^{2 n+1}$ is also embeddable in $\mathbb{C}^{n+1}$ if $2 n+1 \geq 5$, in contrast with the 3-dimensional case (see [Burns and Epstein 1990; Lempert 1992; Nirenberg 1974; Rosay 1979]). Therefore, if $\operatorname{Aut}_{\mathrm{CR}}\left(M, J_{0}\right)$ is noncompact and $2 n+1 \geq 5$, then the situation is reduced to the case of deformations of the unit ball and follows immediately from Theorem 1.2.

The rest of this paper will be devoted to proving Theorem 1.3. Since we are thinking about abstract CR manifolds, we need to develop an intrinsic way of proving this. Therefore, the main interest of Theorem 1.3 is not only in the generalization but also in the intrinsic verification of the Greene-Krantz theorem. The main tool of the proof is the solution for the CR Yamabe problem about the construction of pseudohermitian structures with constant Webster scalar curvature, which is intensively studied in, for instance, [Cheng et al. 2014; Gamara 2001; Gamara and Yacoub 2001; Jerison and Lee 1987; 1989]. The subellipticity of the CR Yamabe equation turned out quite useful in obtaining estimates of derivatives of CR automorphisms in [Schoen 1995]. We make use of various solutions for the CR Yamabe problem - minimal solutions, local scalar flattening solutions and the blowing-up solutions given by the Green functions - developed in [Fischer-Colbrie and Schoen 1980; Jerison and Lee 1987; 1989; Schoen 1995].

\section{Strongly pseudoconvex CR manifolds}

In this section, we summarize fundamental facts on strongly pseudoconvex CR manifolds and pseudohermitian structures. The summation convention is always assumed. 
CR and pseudohermitian structures. Let $M$ be a smooth manifold of dimension $2 n+1$ for some positive integer $n$. A $C R$ structure on $M$ is a smooth complex structure $J$ on a subbundle $H$ of the rank $2 n$ of the tangent bundle $T M$ which satisfies the integrability condition. More precisely, the restriction of $J$ on a fiber $H_{p}$ for a point $p \in M$ is an endomorphism $J_{p}: H_{p} \rightarrow H_{p}$ which satisfies $J_{p} \circ J_{p}=-\operatorname{Id}_{H_{p}}$, varying smoothly as $p$ varies, and the bundle of $i$-eigenspace $H^{1,0}$ of $J$ in the complexification $\mathbb{C} \otimes H$ satisfies the Frobenius integrability condition

$$
\left[\Gamma\left(H^{1,0}\right), \Gamma\left(H^{1,0}\right)\right] \subset \Gamma\left(H^{1,0}\right) .
$$

The subbundle $H$ is called the $C R$ distribution of $J$. A $C R$ automorphism on $M$ is a smooth diffeomorphism $F$ from $M$ onto itself such that $F_{*} H^{1,0}=H^{1,0}$. We denote by $\operatorname{Aut}_{\mathrm{CR}}(M)$ the group of all CR automorphisms on $M$. A CR structure is said to be strongly pseudoconvex if its CR distribution $H$ is a contact distribution and for a contact form $\theta$, the Levi form $\mathcal{L}_{\theta}$ defined by

$$
\mathcal{L}_{\theta}(Z, \bar{W}):=-i d \theta(Z, \bar{W})
$$

for $Z, W \in H^{1,0}$ is positive definite. It is known that the $C^{0}$-topology of $\operatorname{Aut}_{\mathrm{CR}}(M)$ coincides with the $C^{\infty}$-topology for a compact strongly pseudoconvex CR manifold $M$ if $\operatorname{Aut}_{\mathrm{CR}}(M)$ is compact with respect to the $C^{0}$-topology. See [Schoen 1995] for the proof.

We call a fixed contact form for the CR distribution of a strongly pseudoconvex CR structure a pseudohermitian structure. Let $\left\{W_{\alpha}: \alpha=1, \ldots, n\right\}$ be a local frame; that is, the $W_{\alpha}$ are sections of $H^{1,0}$ which form a pointwise basis for $H_{1,0}$. We call a collection of 1-forms $\left\{\theta^{\alpha}\right\}$ the admissible coframe of $\left\{W_{\alpha}\right\}$ if they are sections of $\left(H^{1,0}\right)^{*}$ and satisfy

$$
\theta^{\alpha}\left(W_{\beta}\right)=\delta_{\beta}^{\alpha}, \quad \theta^{\alpha}(T)=0,
$$

where $T$ is the vector field uniquely determined by

$$
\theta(T)=1, \quad T\lrcorner d \theta=0,
$$

which is called the characteristic vector field for $\theta$. Let $g_{\alpha \bar{\beta}}=\mathcal{L}_{\theta}\left(W_{\alpha}, W_{\bar{\beta}}\right)$. Then

$$
d \theta=2 i g_{\alpha \bar{\beta}} \theta^{\alpha} \wedge \theta^{\bar{\beta}},
$$

where $\left\{\theta^{\alpha}\right\}$ is the admissible coframe for $\left\{W_{\alpha}\right\}$.

Theorem 2.1 [Webster 1978]. There exist a local 1-form $\omega=\left(\omega_{\beta}{ }^{\alpha}\right)$ and local functions $A^{\alpha}{ }_{\beta}$ uniquely determined by

$$
\begin{gathered}
d \theta^{\alpha}=\theta^{\beta} \wedge \omega_{\beta}{ }^{\alpha}+A_{\bar{\beta}}^{\alpha} \theta \wedge \theta^{\bar{\beta}}, \\
d g_{\alpha \bar{\beta}}=\omega_{\alpha \bar{\beta}}+\omega_{\bar{\beta} \alpha}, \quad A_{\alpha \beta}=A_{\beta \alpha} .
\end{gathered}
$$


Here and in the sequel, we lower or raise an index by $\left(g_{\alpha \bar{\beta}}\right)$ and $\left(g^{\alpha \bar{\beta}}\right)=\left(g_{\alpha \bar{\beta}}\right)^{-1}$. A connection $\nabla$ defined by

$$
\nabla W_{\alpha}=\omega_{\alpha}^{\beta} \otimes W_{\beta}, \quad \nabla T=0
$$

is called the pseudohermitian connection or the Webster connection for $\theta$. The functions $A^{\alpha}{ }_{\beta}$ are called the coefficients of the torsion tensor $\boldsymbol{T}$. Let

$$
d \omega_{\alpha}{ }^{\beta}-\omega_{\alpha}{ }^{\gamma} \wedge \omega_{\gamma}{ }^{\beta} \equiv R_{\alpha}{ }^{\beta}{ }_{\gamma \bar{\sigma}} \theta^{\gamma} \wedge \theta^{\bar{\sigma}} \bmod \theta, \theta^{\gamma} \wedge \theta^{\sigma}, \theta^{\bar{\gamma}} \wedge \theta^{\bar{\sigma}} .
$$

We call $R_{\alpha}{ }^{\beta}{ }_{\gamma \bar{\sigma}}$ the coefficients of the Webster curvature tensor $\boldsymbol{R}$. Contracting indices, we obtain the coefficients $R_{\alpha \bar{\beta}}$ of the Webster Ricci curvature Ric and the Webster scalar curvature $S$ :

$$
R_{\alpha \bar{\beta}}=R_{\gamma}^{\gamma}{ }_{\alpha \bar{\beta}}, \quad S=R_{\alpha \bar{\beta}} g^{\alpha \bar{\beta}} .
$$

The norm of the Webster curvature $|\boldsymbol{R}|_{\theta}$ is defined by

$$
|\boldsymbol{R}|_{\theta}^{2}=\sum_{\alpha, \beta, \gamma, \sigma}\left|R_{\alpha}{ }^{\beta} \gamma \bar{\sigma}\right|^{2},
$$

where the frame is chosen so that $g_{\alpha \bar{\beta}}=\delta_{\alpha \bar{\beta}}$. We similarly define the norm of the torsion tensor $|\boldsymbol{T}|_{\theta}$.

A pseudohermitian structure defines a sub-Riemannian structure. The distance function induced by a sub-Riemannian metric is called the Carnot-Carathéodory distance (cf. [Strichartz 1986]). We denote by $B_{\theta}(x, r)$ the Carnot-Carathéodory ball with respect to the pseudohermitian structure $\theta$ of radius $r>0$ centered at $x \in M$.

The Heisenberg group $\mathcal{H}^{n}$ is a strongly pseudoconvex CR manifold $\mathbb{C}^{n} \times \mathbb{R}$ with the CR structure whose $H^{1,0}$ bundle is spanned by

$$
Z_{\alpha}=\frac{\partial}{\partial z^{\alpha}}+i z^{\bar{\alpha}} \frac{\partial}{\partial t}, \quad \alpha=1, \ldots, n,
$$

where $(z, t)=\left(z^{1}, \ldots, z^{n}, t\right)$ is the standard coordinate system of $\mathbb{C}^{n} \times \mathbb{R}$. It is well known that $\mathcal{H}^{n}$ is $\mathrm{CR}$ equivalent to the sphere in $\mathbb{C}^{n+1}$ minus a single point. If we put

$$
\vartheta_{0}=d t-i z^{\bar{\alpha}} d z^{\alpha}+i z^{\alpha} d z^{\bar{\alpha}},
$$

then it turns out the curvature and torsion tensors vanish identically. The converse also follows from the solution of the Cartan equivalence problem.

Proposition 2.2. If the curvature and the torsion tensors of a pseudohermitian manifold $(M, \theta)$ vanish identically, then the pseudohermitian structure of $M$ is locally equivalent to that of $\left(\mathcal{H}^{n}, \vartheta_{0}\right)$. If we further assume that $M$ is simply connected and complete in the sense that every Carnot-Carathéodory ball is relatively compact in $M$, then $(M, \theta)$ is globally equivalent to $\left(\mathcal{H}^{n}, \vartheta_{0}\right)$, 
For a given pseudohermitian manifold $(M, \theta)$, we can extend the CR structure $J$ to a smooth section of endomorphism $\hat{J}$ on $T M$ by putting $\hat{J}(T)=0$, where $T$ is the characteristic vector field of $\theta$. Let $J_{k}, k=1,2, \ldots$, and $J_{0}$ be strongly pseudoconvex CR structures on $M$ with CR distributions $H_{k}$ and $H_{0}$, respectively. We say that $J_{k}$ converges to $J_{0}$ in the $C^{l}$ sense $(l=0,1,2, \ldots, \infty)$, if there exist pseudohermitian structures $\theta_{k}$ and $\theta_{0}$ for $\left(M, J_{k}\right)$ and $\left(M, J_{0}\right)$ such that $\theta_{k} \rightarrow \theta_{0}$ and $\hat{J}_{k} \rightarrow \hat{J}_{0}$ in the $C^{l}$ sense as tensors on $M$.

\section{Pseudoconformal change of structures and the CR Yamabe equation. Let $(M, \theta)$} be a $(2 n+1)$-dimensional pseudohermitian manifold and let $\tilde{\theta}=e^{2 f} \theta$ be a pseudoconformal change, where $f$ is a smooth real-valued function. Let $\left\{\theta^{\alpha}\right\}$ be an admissible coframe for $\theta$ satisfying $d \theta=2 i g_{\alpha \bar{\beta}} \theta^{\alpha} \wedge \theta^{\bar{\beta}}$. Then it turns out

$$
\tilde{\theta}^{\alpha}=e^{f}\left(\theta^{\alpha}+i f^{\alpha} \theta\right), \quad \alpha=1, \ldots, n,
$$

form an admissible coframe for $\tilde{\theta}$ which satisfies

$$
d \tilde{\theta}=2 i g_{\alpha \bar{\beta}} \tilde{\theta}^{\alpha} \wedge \tilde{\theta}^{\bar{\beta}} .
$$

Let $R_{\alpha}{ }^{\beta} \gamma \bar{\sigma}$ and $\widetilde{R_{\alpha}{ }^{\beta}} \gamma \bar{\sigma}$ be coefficients of the Webster curvatures for $\theta$ and $\tilde{\theta}$ evaluated in the coframes $\left\{\theta^{\alpha}\right\}$ and $\left\{\tilde{\theta}^{\alpha}\right\}$, respectively. Then they are related as

$$
\begin{aligned}
\widetilde{R_{\alpha}{ }^{\beta} \gamma \bar{\sigma}}=e^{-2 f}\left\{R_{\alpha}{ }^{\beta}{ }_{\gamma \bar{\sigma}}-\right. & \delta_{\alpha}{ }^{\beta}\left(f_{\gamma \bar{\sigma}}+f_{\bar{\sigma} \gamma}\right)-2 g_{\alpha \bar{\sigma}} f^{\beta}{ }_{\gamma}-2 f_{\alpha \bar{\sigma}} \delta^{\beta}{ }_{\gamma} \\
& \left.-\left(f^{\beta}{ }_{\alpha}+f_{\alpha}{ }^{\beta}\right) g_{\gamma \bar{\sigma}}-4\left(\delta_{\alpha}{ }^{\beta} g_{\gamma \bar{\sigma}}+g_{\alpha \bar{\sigma}} \delta^{\beta}{ }_{\gamma}\right) f^{\lambda} f_{\lambda}\right\},
\end{aligned}
$$

where $f_{\alpha \bar{\beta}}, f_{\alpha}{ }^{\beta}$ and $f^{\beta}{ }_{\alpha}$ are components of the second covariant derivatives of $f$ of the pseudohermitian manifold $(M, \theta)$ (cf. Proposition 4.14 in [Joo and Lee 2015] for the more general case). Contracting indices, we obtain the following transformation formula for the Webster scalar curvatures:

$$
\widetilde{S}=e^{-2 f}\left\{S+2(n+1) \Delta_{\theta} f-4 n(n+1) f^{\lambda} f_{\lambda}\right\},
$$

where $\Delta_{\theta} f=-\left(f_{\alpha}{ }^{\alpha}+f_{\bar{\alpha}} \bar{\alpha}^{\bar{\alpha}}\right)$. The operator $\Delta_{\theta}$ is called the sublaplacian for $\theta$.

Let $u$ be a positive smooth function on $M$ defined by $u^{p-2}=e^{2 f}$, where $p=$ $2+2 / n$. Then (2-4) changes into the following nonlinear equation for $u$ :

$$
L_{\theta} u:=\left(b_{n} \Delta_{\theta}+S\right) u=\widetilde{S} u^{p-1},
$$

where $b_{n}=2+2 / n$ (see [Jerison and Lee 1987; 1989; Lee 1986]). Equation (2-5) is called the $C R$ Yamabe equation and the subelliptic linear operator $L_{\theta}$ is called the $C R$ Laplacian for $\theta$. The $C R$ Yamabe problem is to find a positive smooth function $u$ which makes $\widetilde{S}$ constant. 
Let $A^{\alpha}{ }_{\bar{\beta}}$ and ${\widetilde{A^{\alpha}}}_{\bar{\beta}}$ be the coefficients of the torsion tensors for $\theta$ and $\tilde{\theta}$ in the coframes $\left\{\theta^{\alpha}\right\}$ and $\left\{\tilde{\theta}^{\alpha}\right\}$, respectively. Then in turns out that

$$
{\widetilde{A^{\alpha}}}_{\bar{\beta}}=e^{-2 f}\left(A_{\bar{\beta}}^{\alpha}-i f^{\alpha}{ }_{\bar{\beta}}+2 i f^{\alpha} f_{\bar{\beta}}\right) .
$$

See [Lee 1986] for details.

Folland-Stein spaces and subelliptic estimates. Roughly speaking, a normal coordinate system of a pseudohermitian manifold $(M, \theta)$ of dimension $2 n+1$ is a local approximation by the standard pseudohermitian structure on the Heisenberg group $\left(\mathcal{H}^{n}, \theta_{0}\right)$. For $p \in M$, let $W_{1}, \ldots, W_{n}$ be a local frame defined on a neighborhood $V$ of $p$ such that the coefficients of the Levi form for $\theta$ are given by $g_{\alpha \bar{\beta}}=\delta_{\alpha \bar{\beta}}$. Such a frame is called a unitary frame. We denote by $T$ the characteristic vector field for $\theta$. Let $(z, t)$ be the standard coordinates of $\mathcal{H}^{n}$ and let $|(z, t)|=\left(|z|^{4}+t^{2}\right)^{1 / 4}$ be the Heisenberg group norm. We define $Z_{\alpha}$ and $\theta_{0}$ on $\mathcal{H}^{n}$ as (2-1) and (2-2).

Theorem 2.3 [Folland and Stein 1974]. There is a neighborhood of the diagonal $\Omega \subset V \times V$ and $a C^{\infty}$-smooth mapping $\Theta: \Omega \rightarrow \mathcal{H}^{n}$ satisfying:

(a) We have $\Theta(\xi, \eta)=-\Theta(\eta, \xi)=\Theta(\eta, \xi)^{-1}$. (In particular, $\Theta(\xi, \xi)=0$.)

(b) Let $\Theta_{\xi}(\eta)=\Theta(\xi, \eta)$. Then $\Theta_{\xi}$ is a diffeomorphism of a neighborhood $\Omega_{\xi}$ of $\xi$ onto a neighborhood of the origin in $\mathcal{H}^{n}$. Denote by $y=(z, t)=\Theta(\xi, \eta)$ the coordinates of $\mathcal{H}^{n}$. Denote by $O^{k}(k=1,2, \ldots)$ a $C^{\infty}$ function $f$ of $\xi$ and $y$ such that for each compact set $K \subset V$, there is a constant $C_{K}$ with $f(\xi, y) \leq C_{K}|y|^{k}$ (Heisenberg norm) for $\xi \in K$. Then we have the following approximation formula:

$$
\begin{gathered}
\left(\Theta_{\xi}^{-1}\right)^{*} \theta=\theta_{0}+O^{1} d t+\sum_{\alpha=1}^{n}\left(O^{2} d z^{\alpha}+O^{2} d z^{\bar{\alpha}}\right), \\
\left(\Theta_{\xi}^{-1}\right)^{*}\left(\theta \wedge d \theta^{n}\right)=\left(1+O^{1}\right) \theta_{0} \wedge d \theta_{0}^{n}, \\
\Theta_{\xi *} W_{\alpha}=Z_{\alpha}+O^{1} \mathcal{E}\left(\partial_{z}\right)+O^{2} \mathcal{E}\left(\partial_{t}\right), \\
\Theta_{\xi *} T=\partial / \partial t+O^{1} \mathcal{E}\left(\partial_{z}, \partial_{t}\right), \\
\Theta_{\xi *} \Delta_{\theta}=\Delta_{\theta_{0}}+\mathcal{E}\left(\partial_{z}\right)+O^{1} \mathcal{E}\left(\partial_{t}, \partial_{z}^{2}\right)+O^{2} \mathcal{E}\left(\partial_{z} \partial_{t}\right)+O^{3} \mathcal{E}\left(\partial_{t}^{2}\right) .
\end{gathered}
$$

Here $O^{k} \mathcal{E}$ indicates an operator involving linear combinations of the indicated derivatives with smooth coefficients in $O^{k}$, and we have used $\partial_{z}$ to denote any of the derivatives $\partial / \partial z^{\alpha}, \partial / \partial z^{\bar{\alpha}}$.

The smooth map $\Theta_{\xi}$ is called the Folland-Stein normal coordinates centered at $\xi$ with respect to the frame $\left\{W_{\alpha}\right\}$. (This coordinate system depends on the choice of local unitary frame. Another construction of pseudohermitian normal coordinates which does not depend on local frames is given in [Jerison and Lee 1989].) Here 
and in the sequel, we use the term frame constants to mean bounds on finitely many derivatives of the coefficients in the $O^{k} \mathcal{E}$ terms in Theorem 2.3.

Let $V$ be an open neighborhood of a point $p \in M$ with a fixed local unitary frame $W_{1}, \ldots, W_{n}$ and let $U$ be a relatively compact open neighborhood of $p$ in $V$ such that $\Omega_{\xi}$ in Theorem 2.3 contains $\bar{U}$ for every $\xi \in \bar{U}$. Let $X_{\alpha}=\operatorname{Re} W_{\alpha}$ and $X_{\alpha+n}=\operatorname{Im} W_{\alpha}$ for $\alpha=1, \ldots, n$. For a multi-index $A=\left(\alpha_{1}, \ldots, \alpha_{k}\right)$, with $1 \leq \alpha_{j} \leq 2 n, j=1, \ldots, k$, we denote $k$ by $\ell(A)$ and write $X^{A} f=X_{\alpha_{1}} \cdots X_{\alpha_{k}} f$ for a smooth function $f$ on $U$. The $S_{k}^{p}(U)$-norm of a smooth function $f$ on $U$ is

$$
\|f\|_{S_{k}^{p}(U)}=\sup _{\ell(A) \leq k}\left\|X^{A} f\right\|_{L^{p}(U)},
$$

where $\|g\|_{L^{p}(U)}=\left(\int_{U}|g|^{p} \theta \wedge d \theta^{n}\right)^{1 / p}$ is the $L^{p}$-norm of $g$ on $U$ with respect to the volume element induced by $\theta$. The completion of $C_{0}^{\infty}(U)$ with respect to $\|\cdot\|_{S_{k}^{p}(U)}$ is denoted by $S_{k}^{p}(U)$.

Hölder type spaces suited to $\Delta_{\theta}$ are defined as follows. For $x, y \in U$, let $\rho(x, y)=|\Theta(x, y)|$ (Heisenberg norm). For a positive real number $0<s<1$,

$$
\Gamma_{s}(U)=\left\{f \in C^{0}(\bar{U}):|f(x)-f(y)| \leq C \rho(x, y)^{s} \text { for some constant } C>0\right\} .
$$

If $s$ is a positive nonintegral real number such that $k<s<k+1$ for some integer $k \geq 1$, then

$$
\Gamma_{s}(U)=\left\{f \in C^{0}(\bar{U}): X^{A} f \in \Gamma_{s-k}(U), \ell(A) \leq k\right\} .
$$

Then the $\Gamma_{s}(U)$-norm for $f \in \Gamma_{s}(U)$ is defined by

$\|f\|_{\Gamma_{s}(U)}=\sup _{x \in U}|f(x)|+\sup \left\{\frac{\left|X^{A} f(x)-X^{A} f(y)\right|}{\rho(x, y)^{s-k}}: x, y \in U, x \neq y, \ell(A) \leq k\right\}$.

The function spaces $S_{k}^{p}(U)$ and $\Gamma_{s}(U)$ are called the Folland-Stein spaces on $U$. We denote by $\Lambda_{s}(U)$ the Euclidean Hölder space when we regard $U$ as a subset of $\mathbb{R}^{2 n+1}$.

Theorem 2.4 [Folland and Stein 1974]. For each positive real number $s$ which is not an integer, each $1<r<\infty$ and each integer $k \geq 1$, there exists a constant $C>0$ such that for every $f \in C_{0}^{\infty}(U)$,

(a) $\|f\|_{\Gamma_{s}(U)} \leq C\|f\|_{S_{k}^{r}(U)}$, where $1 / r=(k-s) /(2 n+2)$,

(b) $\|f\|_{\Lambda_{s / 2}(U)} \leq C\|f\|_{\Gamma_{s}(U)}$,

(c) $\|f\|_{S_{2}^{r}(U)} \leq C\left(\left\|\Delta_{\theta} f\right\|_{L^{r}(U)}+\|f\|_{L^{r}(U)}\right)$,

(d) $\|f\|_{\Gamma_{s+2}(U)} \leq C\left(\left\|\Delta_{\theta} f\right\|_{\Gamma_{s}(U)}+\|f\|_{\Gamma_{s}(U)}\right)$.

Moreover the constant $C$ depends only on frame constants.

One should notice that the constants $C$ in the theorem above depend on frame constants rather than the pseudohermitian structure itself. Therefore, if $\mathcal{U}$ is a small 
neighborhood $\left(J_{0}, \theta_{0}\right)$ in the $C^{\infty}$-topology, then we can choose constants $C$ in Theorem 2.4 which are independent of the choice of $(J, \theta) \in \mathcal{U}$.

If $M$ is compact, we can choose a finite open covering $U_{1}, \ldots, U_{m}$, each of which is contained in a normal coordinate. Let $\phi_{1}, \ldots, \phi_{m}$ be a partition of unity subordinate to this covering. Then the spaces of $S_{k}^{p}(M)$ and $\Gamma_{s}(M)$ are defined as spaces of a function $u$ such that $\phi_{j} u \in S_{k}^{p}\left(U_{j}\right)$ or $\phi_{j} u \in \Gamma_{s}\left(U_{j}\right)$, respectively, for every $j=1, \ldots, m$.

\section{Proof of Theorem 1.3}

The proof of Theorem 1.3 is based on the following fundamental fact about the semicontinuity property of compact group actions proved by Ebin [1970] for Theorem 1.1. We denote by $\operatorname{Diff}(M)$ the group of $C^{\infty}$-smooth diffeomorphisms. Recall that the $C^{\infty}$-topology on $\operatorname{Diff}(M)$ is metrizable. We denote a metric inducing the $C^{\infty}$-topology by $d$.

Theorem 3.1 ([Ebin 1970]; cf. [Greene et al. 2011; 2013; Grove and Karcher 1973; Kim 1987]). Let $M$ be a compact $C^{\infty}$-smooth manifold and let $G_{k}(k=1,2, \ldots)$ and $G_{0}$ be compact subgroups of $\operatorname{Diff}(M)$. Suppose $G_{j} \rightarrow G_{0}$ in the $C^{\infty}$-topology as $j \rightarrow \infty$; that is, for every $\epsilon>0$, there exists an integer $N$ such that $d\left(f, G_{0}\right):=$ $\inf _{g \in G_{0}} d(f, g)<\epsilon$ for every $f \in G_{j}$, whenever $j>N$. Then $G_{j}$ is isomorphic to a subgroup of $G_{0}$ for every sufficiently large $j$. Moreover, the isomorphism can be obtained by the conjugation by a diffeomorphism $\phi_{j}$ of $M$ which converges to the identity map in the $C^{\infty}$ sense.

Therefore, it suffices to prove the following proposition for the conclusion of Theorem 1.3.

Proposition 3.2. Let $\left\{J_{k}: k=1,2, \ldots\right\}$ be a sequence of strongly pseudoconvex $C R$ structures on a compact manifold $M$ which tends to a strongly pseudoconvex $C R$ structure $J_{0}$ as in Theorem 1.3. Suppose that $\operatorname{Aut}_{\mathrm{CR}}\left(M, J_{0}\right)$ is compact. Then $\operatorname{Aut}_{\mathrm{CR}}\left(M, J_{k}\right)$ is also compact for every sufficiently large $k$. Furthermore, every sequence $\left\{F_{k} \in \operatorname{Aut}_{\mathrm{CR}}\left(M, J_{k}\right): k=1,2, \ldots\right\}$ admits a subsequence converging to an element $F \in \operatorname{Aut}_{\mathrm{CR}}\left(M, J_{0}\right)$ in the $C^{\infty}$ sense.

We will make use of the solutions of the CR Yamabe problem for the proof of Proposition 3.2. According to the variational approach introduced by Jerison and Lee [1987; 1989], it is very natural to consider the sign of the CR Yamabe invariant defined as follows: Let $(M, \theta)$ be a compact pseudohermitian manifold. For a $C^{\infty}$-smooth real-valued function $u$, let

$$
A(\theta ; u):=\int_{M} u L_{\theta} u \theta \wedge d \theta^{n}=\int_{M}\left(b_{n}|d u|_{\theta}^{2}+R u^{2}\right) \theta \wedge d \theta^{n}
$$


and

$$
B(\theta ; u):=\int_{M}|u|^{p} \theta \wedge d \theta^{n} .
$$

Then the $C R$ Yamabe invariant $Y(M)$ is defined by

$$
Y(M):=\inf \left\{A(\theta ; u): u \in C^{\infty}(M), B(\theta ; u)=1\right\} .
$$

It is well known that $Y(M)$ does not depend on the choice of contact form $\theta$. Let $J_{k}$ be a sequence of strongly pseudoconvex CR structures on $M$ tending to a strongly pseudoconvex CR structure $J_{0}$ as $k \rightarrow \infty$. We denote by $Y_{k}$ the CR Yamabe invariant of $\left(M, J_{k}\right)$. For the proof, we may assume either that $Y_{k} \leq 0$ for every $k$ or that $Y_{k}>0$ for every $k$.

Case $\boldsymbol{Y}_{\boldsymbol{k}} \leq \mathbf{0}$. In this case, we use the minimal solution of the Yamabe problem.

Theorem 3.3 [Jerison and Lee 1987]. Let $M$ be a compact strongly pseudoconvex CR manifold of dimension $2 n+1$.

(i) $Y\left(S^{2 n+1}\right)>0$, where $Y\left(S^{2 n+1}\right)$ is the CR Yamabe invariant for the sphere $S^{2 n+1}$ with the standard structure.

(ii) $Y(M) \leq Y\left(S^{2 n+1}\right)$.

(iii) If $Y(M)<Y\left(S^{2 n+1}\right)$, then there exists a positive $C^{\infty}$-smooth function $u$ which satisfies $B(\theta ; u)=1$ and $A(\theta ; u)=Y(M)$ for a given pseudohermitian structure $\theta$. This function $u$ satisfies

$$
L_{\theta} u=Y(M) u^{p-1} .
$$

That is, the pseudohermitian structure $\tilde{\theta}=u^{p-2} \theta$ has a constant Webster scalar curvature $\widetilde{R}=Y(M)$.

It is known from [Jerison and Lee 1989] that $Y(M)<Y\left(S^{2 n+1}\right)$ if $M$ is not locally spherical and $2 n+1 \geq 5$. The cases that $2 n+1=3$ or that $M$ is spherical are dealt with in [Gamara 2001; Gamara and Yacoub 2001].

Proposition 3.4 [Jerison and Lee 1987, Theorem 7.1]. If $Y(M) \leq 0$, then a pseudohermitian structure with constant Webster scalar curvature is unique up to constant multiples. As a consequence, there is a unique pseudohermitian structure with constant Webster scalar curvature under the unit volume condition, if $Y(M) \leq 0$.

Proposition 3.5 [Jerison and Lee 1987, Theorem 5.15]. Let $M$ be a compact strongly pseudoconvex $C R$ manifold of dimension $2 n+1$ and let $\theta$ be a pseudohermitian structure. Suppose that $f, g \in C^{\infty}(M), u \geq 0, u \in L^{r}$ for some $r>p=2+2 / n$ and

$$
\Delta_{\theta} u+g u=f u^{q-1}
$$


in the distribution sense for some $2 \leq q \leq p$. Then $u \in C^{\infty}(M), u>0$. Furthermore, $\|u\|_{C^{k}}$ depends only on $\|u\|_{L^{r}},\|f\|_{C^{k}},\|g\|_{C^{k}}$ and frame constants, but not on $q$.

Indeed, a local version of the above lemma is stated in [Jerison and Lee 1987]. But it is obvious it holds globally by taking a partition of unity subordinate to a chart of normal coordinates.

Proposition 3.6 [Jerison and Lee 1987, Proposition 5.5, case $k=1, r=2$ and $s=p]$. For a compact pseudohermitian manifold $(M, \theta)$ of dimension $2 n+1$, there exists a constant $C>0$ such that

$$
\int_{M}|v|^{p} \theta \wedge d \theta^{n} \leq C \int_{M}\left(|d v|_{\theta}^{2}+|v|^{2}\right) \theta \wedge d \theta^{n}
$$

for every $C^{\infty}$-smooth function $v$ on $M$.

Since we are considering CR structures converging to the target structure $J_{0}$, we can choose also a sequence $\left\{\theta_{k}\right\}$ of contact forms which tends to a target pseudohermitian structure $\theta_{0}$ in the $C^{\infty}$ sense. Without loss of generality, we always assume that $\int_{M} \theta_{k} \wedge d \theta_{k}^{n}=1$ for every $k$.

Lemma 3.7. Suppose that $Y_{k} \leq 0$ for every $k$. Let $u_{k}>0$ be the (unique) solution as in Theorem 3.3(iii) with respect to $\left(J_{k}, \theta_{k}\right)$. Then for each nonnegative integer $l$, there exists a constant $C$ such that $\left\|u_{k}\right\|_{C^{l}} \leq C$ for every $k$.

Proof. Since $u_{k}$ satisfies

$$
b_{n} \Delta_{\theta_{k}} u_{k}+R_{k} u_{k}=Y_{k} u_{k}^{p-1}
$$

where $R_{k}$ is the Webster scalar curvature for $\theta_{k}$, we have

$$
\int_{M} \frac{1}{2}(p-1) b_{n} u_{k}^{p-2}\left|d u_{k}\right|_{\theta_{k}} \theta_{k} \wedge d \theta_{k}^{n} \leq \int_{M}\left|R_{k} u_{k}^{p}\right| \theta_{k} \wedge d \theta_{k}^{n}
$$

by integrating after multiplying by $u_{k}^{p-1}$ on both sides of (3-1), since $Y_{k} \leq 0$. Therefore, the function $w_{k}:=u_{k}^{p / 2}$ satisfies

$$
\int_{M}\left|d w_{k}\right|_{\theta_{k}}^{2} \theta_{k} \wedge d \theta_{k}^{n} \leq C \int_{M} w_{k}^{2} \theta_{k} \wedge d \theta_{k}^{n}=C \int_{M} u_{k}^{p} \theta_{k} \wedge d \theta_{k}^{n}=C,
$$

since $R_{k}$ is bounded uniformly for $k$. Moreover since $\left(J_{k}, \theta_{k}\right) \rightarrow\left(J_{0}, \theta_{0}\right)$ in the $C^{\infty}$ sense, Proposition 3.6 implies that there exists a constant $C>0$ independent of $k$ such that

$$
\int_{M} w_{k}^{p} \theta_{k} \wedge d \theta_{k}^{n} \leq C \int_{M}\left(\left|d w_{k}\right|_{\theta_{k}}^{2}+w_{k}^{2}\right) \theta_{k} \wedge d \theta_{k}^{n},
$$

which is uniformly bounded for every $k$. This implies that $\left\|u_{k}\right\|_{L^{r}}$ is uniformly bounded as $\left(J_{k}, \theta_{k}\right) \rightarrow\left(J_{0}, \theta_{0}\right)$, where $r=\frac{1}{2} p^{2}>p$. Then the conclusion follows from Proposition 3.5, since frame constants for $\left(J_{k}, \theta_{k}\right)$ are also uniformly bounded as $\left(J_{k}, \theta_{k}\right) \rightarrow\left(J_{0}, \theta_{0}\right)$ in the $C^{\infty}$ sense. 
If $Y_{k} \leq 0$ for every $k \geq 1$, then by taking a subsequence, we may assume the sequence $\left\{u_{k}\right\}$ of solutions of the Yamabe problem with respect to $\left(J_{k}, \theta_{k}\right)$ converges to $u_{0}$, the solution of the Yamabe problem with respect to $\left(J_{0}, \theta_{0}\right)$ in the $C^{\infty}$ sense by Lemma 3.7. Replacing $\theta_{k}$ by $u_{k}^{p-2} \theta_{k}$, then we may assume the Webster scalar curvature of $\theta_{k}$ is a nonpositive constant for every $k$. In this case, it is known that the CR automorphism group of $\left(M, J_{k}\right)$ coincides with the pseudohermitian automorphism group for $\left(M, J_{k}, \theta_{k}\right)$. Let $g_{k}$ be the Riemannian metric on $M$ defined by

$$
g_{k}=\theta_{k} \otimes \theta_{k}+d \theta_{k}\left(\cdot, J_{k} \cdot\right)
$$

for each $k$. Then we see that $g_{k} \rightarrow g_{0}=\theta_{0} \otimes \theta_{0}+d \theta_{0}\left(\cdot, J_{0} \cdot\right)$ in the $C^{\infty}$ sense, and the CR automorphism groups $\operatorname{Aut}_{\mathrm{CR}}\left(M, J_{k}\right)$ and $\operatorname{Aut}_{\mathrm{CR}}\left(M, J_{0}\right)$ are subgroups of the isometry groups of $g_{k}$ and $g_{0}$, respectively. Then the conclusion follows from the proof of Theorem 1.1.

Case $\boldsymbol{Y}_{\boldsymbol{k}}>\mathbf{0}$. We will show that if a sequence $\left\{F_{k} \in \operatorname{Aut}_{\mathrm{CR}}\left(M, J_{k}\right)\right\}$ is divergent, then it generates a single "bubble" which is CR equivalent to $\left(M, J_{0}\right)$. This case should be excluded by proving the CR structure of the bubble is the same as that of the standard sphere, which contradicts the hypothesis that $\operatorname{Aut}_{\mathrm{CR}}\left(M, J_{0}\right)$ is compact. An essential ingredient for analyzing the bubbling phenomenon is the reparametrization of the pseudohermitian structure by the Green function of the CR Laplacian. The existence of the Green function is guaranteed by the hypothesis $Y_{k}>0$ (see, for instance, [Cheng et al. 2014; Gamara 2001]). We discuss the bubbling after the following fundamental lemma on the convergence of CR automorphisms.

Lemma 3.8. Suppose for a sequence $\left\{F_{k} \in \operatorname{Aut}_{\mathrm{CR}}\left(M, J_{k}\right)\right\}, F_{k} \rightarrow F$ and $F_{k}^{-1} \rightarrow G$ in the $C^{0}$ sense for some continuous mappings $F$ and $G$. Then $F \in \operatorname{Aut}_{\mathrm{CR}}\left(M, J_{0}\right)$, $G=F^{-1}$ and $F_{k} \rightarrow F$ in the $C^{\infty}$ sense.

Proof. This lemma is a sequential version of Proposition 1.1' in [Schoen 1995]. Let $\theta_{k}$ and $\theta_{0}$ be pseudohermitian structures for $J_{k}$ and $J_{0}$, respectively, and suppose $\theta_{k} \rightarrow \theta_{0}$ in the $C^{\infty}$ sense. For a given point $p \in M$, let $q_{k}=F_{k}(p)$ and $q=F(p)$. Let $q \in \widetilde{U} \Subset \widetilde{V} \Subset \widetilde{W}$ be relatively compact neighborhoods of $q$. Since $q_{k} \rightarrow q$, we can assume that $q_{k} \in \widetilde{U}$ for every $k$. The fact that $Y_{k}>0$ implies that the principal eigenvalue of $L_{\theta_{k}}$ on $M$, and hence the Dirichlet principal eigenvalue of $L_{\theta_{k}}$ on $\widetilde{W}$, is also positive for every $k$. Then by the local scalar flattening argument of FischerColbrie and Schoen [1980; 1995], we have a positive $C^{\infty}$-smooth function $u_{k}$ on $\widetilde{W}$ such that $L_{\theta_{k}} u_{k}=0$ on $\widetilde{W}$ for every $k$. Multiplying by a positive constant, we may assume that $u_{k}(q)=1$ for every $k$. Then the subelliptic theory in Theorem 2.3 for the sublaplacian and the Harnack principle (cf. Proposition 5.12 in [Jerison and Lee 1987]) imply that $\left\{u_{k}\right\}$ has a convergent subsequence which tends to a positive function $u_{0}$ on the closure of $\widetilde{V}$ in the $C^{\infty}$ sense. We denote the convergent subsequence by $\left\{u_{k}\right\}$ again. Then $\tilde{\theta}_{k}=u_{k}^{p-2} \theta_{k}$ and $\tilde{\theta}_{0}=u_{0}^{p-2} \theta_{0}$ have the trivial 
Webster scalar curvatures on $\widetilde{V}$. From the equicontinuity of the sequence $\left\{F_{k}\right\}$, we can choose a neighborhood $W$ of $p$ such that $F_{k}(W) \in \widetilde{U}$ for every $k$. Let $v_{k}$ be a positive smooth function on $V$ defined by $F_{k}^{*} \tilde{\theta}_{k}=v_{k}^{p-2} \theta_{k}$. Then for every $k$, we have

$$
L_{\theta_{k}} v_{k}=0 \quad \text { on } W .
$$

We denote by $\operatorname{Vol}_{\tilde{\theta}_{k}}(\tilde{U})$ the volume of $\widetilde{U}$ with respect to the volume form $\tilde{\theta}_{k} \wedge d \tilde{\theta}_{k}^{n}$. Since $\tilde{\theta}_{k}$ converges to $\tilde{\theta}_{0}$ in the $C^{\infty}$ sense in $\widetilde{V}$, there exists a uniform bound $C$ of $\operatorname{Vol}_{\tilde{\theta}_{k}}(\widetilde{U})$. Therefore, it turns out that

$$
\int_{W} v_{k}^{p} \theta_{k} \wedge d \theta_{k}^{n}=\int_{W} F_{k}^{*}\left(\tilde{\theta}_{k} \wedge d \tilde{\theta}_{k}\right)=\operatorname{Vol}_{\tilde{\theta}_{k}}\left(F_{k}(W)\right) \leq \operatorname{Vol}_{\tilde{\theta}_{k}}(\widetilde{U}) \leq C
$$

for every $k$. Fix a neighborhood $V \Subset W$ of $p$. Then the subelliptic mean-value inequality for (3-2) implies that there exists a constant $C$ such that $v_{k}(x) \leq C$ for every $x \in V$. We can also choose this $C$ independently on $k$ by the convergence of structures. Then for a given neighborhood $U \Subset V$ of $p$ and for each positive integer $l$, there exists a constant $C_{l}$ which is independent of $k$ such that

$$
\left\|v_{k}\right\|_{C^{l}(U)} \leq C_{l}
$$

for every $k$, by Theorem 2.3. Since each $F_{k}$ is pseudoconformal, the $C^{l}$-norm of $F_{k}$ on $U$ is completely determined by that of $v_{k}$ and is uniformly bounded on $U$. This yields that every subsequence of $\left\{F_{k}\right\}$ contains a subsequence converging in the $C^{l}$ sense, for every positive integer $l$. Since $F_{k}$ converges to $F$ in the $C^{0}$ sense on $M$ and since $M$ is compact, we conclude that $F_{k}$ converges to $F$ in the $C^{\infty}$ sense. By the same reasoning, $F_{k}^{-1} \rightarrow G$ in the $C^{\infty}$ sense. It follows immediately that $F \in \operatorname{Aut}_{\mathrm{CR}}\left(M, J_{0}\right)$ and $G=F^{-1}$.

For a CR diffeomorphism $F:(M, \theta) \rightarrow(\tilde{M}, \tilde{\theta})$ between two pseudohermitian manifolds, we denote by $\left|F^{\prime}\right|_{\theta, \tilde{\theta}}$ the pseudoconformal factor of $F$, that is, $F^{*} \tilde{\theta}=$ $\left|F^{\prime}\right|_{\theta, \tilde{\theta}} \theta$. We abbreviate it to $\left|F^{\prime}\right|_{\theta}$ in case $(M, \theta)=(\tilde{M}, \tilde{\theta})$.

Lemma 3.9. Let $(M, \theta)$ and $(\tilde{M}, \tilde{\theta})$ be pseudohermitian manifolds of the same dimension. Let $K$ be a relatively compact subset of $M$ and suppose that the Webster scalar curvature for $\tilde{\theta}$ vanishes on $\tilde{M}$. Then there exist constants $r_{0}>0$ and $C>0$ such that for every $C R$ diffeomorphism $F$ on a Carnot-Carathéodory ball $B_{\theta}(x, r)$ into $M$,

$$
B_{\tilde{\theta}}\left(F(x), C^{-1} \lambda r\right) \subset F\left(B_{\theta}(x, r)\right) \subset B_{\tilde{\theta}}(F(x), C \lambda r)
$$

whenever $x \in K$ and $r \leq \frac{1}{2} r_{0}$, where $\lambda=\left|F^{\prime}\right|_{\theta, \tilde{\theta}}(x)$. The constant $C$ depends only on $r_{0}, K$ and uniform bounds of finite-order derivatives of the $C R$ and pseudohermitian structures of $(M, \theta)$.

This lemma is a restatement of Proposition 2.1'(i) in [Schoen 1995], which is a consequence of the subelliptic Harnack principle. 
To prove Proposition 3.2, assume the contrary. Then there exists a sequence $\left\{F_{k} \in \operatorname{Aut}_{\mathrm{CR}}\left(M, J_{k}\right)\right\}$ such that $\sup _{x \in M}\left|F_{k}^{\prime}\right|_{\theta_{k}}(x) \rightarrow \infty$ as $k \rightarrow \infty$, thanks to Lemma 3.8. Let $x_{k} \in M$ be a point of $M$ with $\left|F_{k}^{\prime}\right|_{\theta_{k}}\left(x_{k}\right)=\sup _{x \in M}\left|F_{k}^{\prime}\right|_{\theta_{k}}(x)$. Extracting a subsequence, we assume that $x_{k} \rightarrow x_{0} \in M$ and $F_{k}\left(x_{k}\right) \rightarrow z_{0}$ as $k \rightarrow \infty$. Choose $r>0$ small enough that the Carnot-Carathéodory balls satisfy $B_{\theta_{k}}\left(x_{k}, r\right) \Subset B_{\theta_{k}}\left(x_{k}, 2 r\right) \Subset U$ for each $k$, where $U$ is a relatively compact neighborhood of $x_{0}$ in $M$, and $2 r<r_{0}$ for $r_{0}$ given in Lemma 3.9.

Lemma 3.10. There exists a subsequence $\left\{F_{k_{j}}: j=1,2, \ldots\right\}$ of $\left\{F_{k}: k=1,2, \ldots\right\}$ which admits a point $y_{0} \in M$ such that for every compact subset $K$ in $M \backslash\left\{y_{0}\right\}$, there exists $N>0$ such that $K \subset F_{k_{j}}\left(B_{\theta_{k_{j}}}\left(x_{k_{j}}, 2 r\right)\right)$ if $k_{j}>N$. Moreover, for the subsequence, one can choose the point $y_{0}$ independently of $r>0$ as $r \rightarrow 0$.

Proof. Suppose for every $r>0$, there exists no sequence $\left\{y_{k} \in M \backslash F_{k}\left(B_{\theta_{k}}\left(x_{k}, 2 r\right)\right)\right\}$ such that $d\left(y_{k}, F_{k}\left(x_{k}\right)\right)>\epsilon$ for any given $\epsilon>0$, where $d$ is the sub-Riemannian distance induced from $\theta_{0}$. Then it turns out every sequence $\left\{y_{k} \in M \backslash F_{k}\left(B_{\theta_{k}}\left(x_{k}, 2 r\right)\right)\right\}$ converges to $z_{0}$. In this case, we just need to put $y_{0}=z_{0}$.

Now suppose for some $r>0$, there exists a sequence $\left\{y_{k} \in M \backslash F_{k}\left(B_{\theta_{k}}\left(x_{k}, 2 r\right)\right)\right\}$ such that $d\left(y_{k}, F_{k}\left(x_{k}\right)\right)>\epsilon$ for infinitely many $k$ for some $\epsilon>0$. Extracting a subsequence, we may assume that $y_{k} \rightarrow y_{0} \in M$ and $d\left(y_{k}, F_{k}\left(x_{k}\right)\right)>\epsilon$ for every $k$ so that the sequence $\left\{F_{k}\left(x_{k}\right)\right\}$ is relatively compact in $M \backslash\left\{y_{0}\right\}$. Let $G_{k}$ be the Green function for $L_{\theta_{k}}$ with pole at $y_{k}$. We normalize $G_{k}$ by the condition $\min _{M \backslash\left\{y_{k}\right\}} G_{k}=1$. Since each $G_{k}>0$ and $L_{\theta_{k}} G_{k}=0$ on $M \backslash\left\{y_{k}\right\}$, we may assume $\left\{G_{k}: k=1,2, \ldots\right\}$ converges to a positive function $G_{0}$ on $M \backslash\left\{y_{0}\right\}$ in the local $C^{\infty}$ sense, by extracting a subsequence if necessary. Let $\tilde{\theta}_{k}=G_{k}^{p-2} \theta_{k}$. Then $\tilde{\theta}_{k}$ is a pseudohermitian structure on $M \backslash\left\{y_{k}\right\}$ which is Webster scalar flat. Therefore, if we denote $\lambda_{k}=\left|F_{k}^{\prime}\right|_{\theta_{k}, \tilde{\theta}_{k}}\left(x_{k}\right)$, then Lemma 3.9 implies that there exists a constant $C$ independent of $k$ such that

$$
B_{\tilde{\theta}_{k}}\left(F_{k}\left(x_{k}\right), C^{-1} \lambda_{k} r\right) \subset F_{k}\left(B_{\theta_{k}}\left(x_{k}, r\right)\right) \subset B_{\tilde{\theta}_{k}}\left(F_{k}\left(x_{k}\right), C \lambda_{k} r\right) .
$$

Since $G_{k} \geq 1$ and $\left|F_{k}^{\prime}\right|_{\theta_{k}}\left(x_{k}\right) \rightarrow \infty, \lambda_{k}$ also tends to infinity as $k \rightarrow \infty$. Therefore, a relatively compact subset $K$ in $M \backslash\left\{y_{0}\right\}$ should be included in $F_{k}\left(B_{\theta_{k}}\left(x_{k}, r\right)\right.$ ) for every sufficiently large $k$, since $F_{k}\left(x_{k}\right)$ lies on a fixed relatively compact subset of $M \backslash\left\{y_{0}\right\}$ and $\tilde{\theta}_{k} \rightarrow \tilde{\theta}_{0}=G_{0}^{p-2} \theta_{0}$ in the local $C^{\infty}$-smooth sense on $M \backslash\left\{y_{0}\right\}$. Note that the choice of the sequence $\left\{y_{k}\right\}$ and $y_{0}$ still works for every $r^{\prime} \leq r$. This yields the independence of $y_{0}$ on $r$ as $r \rightarrow 0$.

As a consequence of Lemma 3.10, it turns out that $M \backslash\left\{y_{0}\right\}$ is simply connected and complete with respect to the sub-Riemannian distance induced by $\tilde{\theta}_{0}$. In fact, any loop in $M \backslash\left\{y_{0}\right\}$ is contained in $F_{k}\left(B_{\theta_{k}}\left(x_{k}, 2 r\right)\right)$ for some large $k$ by Lemma 3.10. Since $B_{\theta_{k}}\left(x_{k}, 2 r\right)$ is simply connected if $r>0$ is small enough and since $F_{k}$ is a 
diffeomorphism, $F_{k}\left(B_{\theta_{k}}\left(x_{k}, 2 r\right)\right)$ is simply connected as well. Therefore, the given loop should be contractible. This shows that $M \backslash\left\{y_{0}\right\}$ is simply connected.

Extracting a subsequence, we assume that Lemma 3.10 holds for the entire sequence $\left\{F_{k}\right\}$. Choose $y_{k} \in M \backslash F_{k}\left(B_{\theta_{k}}\left(x_{k}, 2 r\right)\right)$ which tends to $y_{0}$. Let $v_{k}$ and $f_{k}$ be real-valued functions on $B_{\theta_{k}}\left(x_{k}, 2 r\right)$ defined by

$$
v_{k}^{p-2}=\left|F_{k}^{\prime}\right|_{\theta_{k}, \tilde{\theta}_{k}}=e^{2 f_{k}},
$$

where $G_{k}$ is the normalized Green function for $L_{\theta_{k}}$ with pole at $y_{k}$ which converges to a positive function $G_{0}$ in the local $C^{\infty}$-smooth sense on $M \backslash\left\{y_{0}\right\}$ as $k \rightarrow \infty$, and $\tilde{\theta}_{k}=G_{k}^{p-2} \theta_{k}$. Since $L_{\theta_{k}} v_{k}=0$, we see that there exists a constant $C$ independent of $k$ such that $\left|F_{k}^{\prime}\right|_{\theta_{k}, \tilde{\theta}_{k}} \geq C \lambda_{k}$ on $B_{\theta_{k}}\left(x_{k}, r\right)$ by the Harnack principle, where $\lambda_{k}=$ $\left|F_{k}^{\prime}\right|_{\theta_{k}, \tilde{\theta}_{k}}\left(x_{k}\right)$. Let $\left\{Z_{k} \in \Gamma\left(H_{k}^{1,0}\right)\right\}$ be a sequence of vector fields on $U$ which tends to $Z_{0} \in \Gamma\left(H_{0}^{1,0}\right)$ as $k \rightarrow \infty$, where $H_{k}^{1,0}$ represents the $(1,0)$-bundle with respect to $J_{k}$. Since $f_{k}=(1 / n) \log v_{k}$, we have

$$
Z_{k} f_{k}=\frac{Z_{k} v_{k}}{n v_{k}}
$$

for every $k$. Since $L_{\theta_{k}} v_{k}=0$ on $B_{\theta_{k}}\left(x_{k}, 2 r\right)$, the subelliptic estimates in Theorem 2.4 imply that $Z_{k} f_{k}$ is uniformly bounded on $B_{\theta_{k}}\left(x_{k}, r\right)$ for every $k$. So is $\bar{Z}_{k} f_{k}$, and if $W_{k}$ is another sequence of vector fields, then $Z_{k} W_{k} f_{k}$ and $Z_{k} \bar{W}_{k} f_{k}$ are all uniformly bounded on $B_{\theta_{k}}\left(x_{k}, r\right)$ as $k \rightarrow \infty$. Therefore, if we denote by $\boldsymbol{R}_{k}$ and $\widetilde{\boldsymbol{R}}_{k}$ the Webster curvature tensors for $\theta_{k}$ and $\tilde{\theta}_{k}$, respectively, then (2-3) implies that

$$
\left|\widetilde{\boldsymbol{R}}_{k}\right|_{\tilde{\theta}_{k}}^{2}\left(F_{k}(x)\right) \leq C \lambda_{k}^{-2}\left\{\left|\boldsymbol{R}_{k}\right|_{\theta_{k}}^{2}(x)+A_{k}\left|\boldsymbol{R}_{k}\right|_{\theta_{k}}(x)+B_{k}\right\}
$$

for every $x \in B_{\theta_{k}}\left(x_{k}, r\right)$, where $A_{k}$ and $B_{k}$ are some functions of the first and second covariant derivatives of $f_{k}$ with respect to the pseudohermitian structure $\theta_{k}$ which are uniformly bounded on $B_{\theta_{k}}\left(x_{k}, r\right)$ as $k \rightarrow \infty$. Since $\lambda_{k} \rightarrow \infty$ and $\left|\boldsymbol{R}_{k}\right|_{\theta_{k}}$ is uniformly bounded on $B_{\theta_{k}}\left(x_{k}, r\right)$ for every $k$, it turns out that $\left|\widetilde{\boldsymbol{R}}_{k}\right|_{\tilde{\theta}_{k}} \rightarrow 0$ uniformly on every compact subset of $M \backslash\left\{y_{0}\right\}$ by Lemma 3.10. Therefore, we see that the pseudohermitian manifold $\left(M \backslash\left\{y_{0}\right\}, \tilde{\theta}_{0}\right)$ has trivial Webster curvature. A similar argument using (2-6) implies that the torsion tensor of $\tilde{\theta}_{0}$ is also trivial. Therefore, we can conclude that $\left(M \backslash\left\{y_{0}\right\}, \tilde{\theta}_{0}\right)$ is equivalent to the standard pseudohermitian structure of the Heisenberg group and therefore, $\left(M, J_{0}\right)$ is CR equivalent to the sphere by the removable singularity theorem. This contradicts the hypothesis that $\operatorname{Aut}_{\mathrm{CR}}\left(M, J_{0}\right)$ is compact and hence yields the conclusion of Proposition 3.2.

\section{References}

[Bell 1987] S. Bell, "Compactness of families of holomorphic mappings up to the boundary", pp. 29-42 in Complex analysis (University Park, PA, 1986), edited by S. G. Krantz, Lecture Notes in Mathematics 1268, Springer, Berlin, 1987. MR 907051 Zbl 0633.32020 
[Boutet de Monvel 1975] L. Boutet de Monvel, "Intégration des équations de Cauchy-Riemann induites formelles", Exposé No. 9 in Séminaire Goulaouic-Lions-Schwartz. 1974-1975; équations aux derivées partielles linéaires et non linéaires, Centre Math., École Polytech., Paris, 1975. MR 0409893 Zbl 0317.58003

[Burns and Epstein 1990] D. M. Burns and C. L. Epstein, "Embeddability for three-dimensional CR-manifolds", J. Amer. Math. Soc. 3:4 (1990), 809-841. MR 1071115 Zbl 0736.32017

[Cheng et al. 2014] J.-H. Cheng, H.-L. Chiu, and P. Yang, "Uniformization of spherical CR manifolds", Adv. Math. 255 (2014), 182-216. MR 3167481 Zbl 1288.32051

[Ebin 1970] D. G. Ebin, "The manifold of Riemannian metrics", pp. 11-40 in Global analysis (Berkeley, CA, 1968), edited by S.-S. Chern and S. Smale, Proceedings of Symposia in Pure Mathematics 15, Amer. Math. Soc., Providence, RI, 1970. MR 0267604 Zbl 0205.53702

[Fefferman 1974] C. Fefferman, "The Bergman kernel and biholomorphic mappings of pseudoconvex domains”, Invent. Math. 26 (1974), 1-65. MR 0350069 Zbl 0289.32012

[Fischer-Colbrie and Schoen 1980] D. Fischer-Colbrie and R. Schoen, "The structure of complete stable minimal surfaces in 3-manifolds of nonnegative scalar curvature", Comm. Pure Appl. Math. 33:2 (1980), 199-211. MR 562550 Zbl 0439.53060

[Folland and Stein 1974] G. B. Folland and E. M. Stein, "Estimates for the $\bar{\partial}_{b}$ complex and analysis on the Heisenberg group", Comm. Pure Appl. Math. 27 (1974), 429-522. MR 0367477 Zbl 0293.35012

[Gamara 2001] N. Gamara, "The CR Yamabe conjecture - the case $n=1$ ", J. Eur. Math. Soc. (JEMS) 3:2 (2001), 105-137. MR 1831872 Zbl 0988.53013

[Gamara and Yacoub 2001] N. Gamara and R. Yacoub, "CR Yamabe conjecture - the conformally flat case”, Pacific J. Math. 201:1 (2001), 121-175. MR 1867895 Zbl 1054.32020

[Greene and Kim 2014] R. E. Greene and K.-T. Kim, "Stably-interior points and the semicontinuity of the automorphism group”, Math. Z. 277:3-4 (2014), 909-916. MR 3229971 Zbl 06323341

[Greene and Krantz 1982] R. E. Greene and S. G. Krantz, "The automorphism groups of strongly pseudoconvex domains”, Math. Ann. 261:4 (1982), 425-446. MR 682655 Zbl 0531.32016

[Greene et al. 2011] R. E. Greene, K.-T. Kim, and S. G. Krantz, The geometry of complex domains, Progress in Mathematics 291, Birkhäuser, Boston, 2011. MR 2799296 Zbl 1239.32011

[Greene et al. 2013] R. E. Greene, K.-T. Kim, S. G. Krantz, and A. Seo, "Semicontinuity of automorphism groups of strongly pseudoconvex domains: the low differentiability case", Pacific J. Math. 262:2 (2013), 365-395. MR 3069066 Zbl 1271.32021

[Grove and Karcher 1973] K. Grove and H. Karcher, "How to conjugate $C^{1}$-close group actions", Math. Z. 132 (1973), 11-20. MR 0356104 Zbl 0245.57016

[Hamilton 1977] R. S. Hamilton, "Deformation of complex structures on manifolds with boundary, I: The stable case", J. Differential Geometry 12:1 (1977), 1-45. MR 0477158 Zbl 0394.32010

[Hamilton 1979] R. S. Hamilton, "Deformation of complex structures on manifolds with boundary, II: Families of noncoercive boundary value problems", J. Differential Geom. 14:3 (1979), 409-473. MR 594711 Zbl 0512.32015

[Jerison and Lee 1987] D. Jerison and J. M. Lee, "The Yamabe problem on CR manifolds", J. Differential Geom. 25:2 (1987), 167-197. MR 880182 Zbl 0661.32026

[Jerison and Lee 1989] D. Jerison and J. M. Lee, "Intrinsic CR normal coordinates and the CR Yamabe problem”, J. Differential Geom. 29:2 (1989), 303-343. MR 982177 Zbl 0671.32016

[Joo and Lee 2015] J.-C. Joo and K.-H. Lee, "Subconformal Yamabe equation and automorphism groups of almost CR manifolds", J. Geom. Anal. 25:1 (2015), 436-470. MR 3299289 Zbl 1310.32038 
[Kim 1987] Y. W. Kim, "Semicontinuity of compact group actions on compact differentiable manifolds", Arch. Math. (Basel) 49:5 (1987), 450-455. MR 915919 Zbl 0615.57020

[Krantz 2010] S. G. Krantz, "Convergence of automorphisms and semicontinuity of automorphism groups”, Real Anal. Exchange 36:2 (2010), 421-433. MR 3016726 Zbl 1271.32018

[Lee 1986] J. M. Lee, "The Fefferman metric and pseudo-Hermitian invariants", Trans. Amer. Math. Soc. 296:1 (1986), 411-429. MR 837820 Zbl 0595.32026

[Lempert 1992] L. Lempert, "On three-dimensional Cauchy-Riemann manifolds", J. Amer. Math. Soc. 5:4 (1992), 923-969. MR 1157290 Zbl 0781.32014

[Nirenberg 1974] L. Nirenberg, "A certain problem of Hans Lewy”, Uspehi Mat. Nauk 29:2(176) (1974), 241-251. In Russian; translated in Russian Math. Surveys 29:2 (1974), 251-262. MR 0492752 Zbl 0306.35019

[Rosay 1979] J.-P. Rosay, "Sur une caractérisation de la boule parmi les domaines de $\mathbf{C}^{n}$ par son groupe d'automorphismes", Ann. Inst. Fourier (Grenoble) 29:4 (1979), ix, 91-97. MR 558590 Zbl 0402.32001

[Schoen 1995] R. Schoen, "On the conformal and CR automorphism groups", Geom. Funct. Anal. 5:2 (1995), 464-481. MR 1334876 Zbl 0835.53015

[Strichartz 1986] R. S. Strichartz, "Sub-Riemannian geometry", J. Differential Geom. 24:2 (1986), 221-263. MR 862049 Zbl 0609.53021

[Webster 1978] S. M. Webster, "Pseudo-Hermitian structures on a real hypersurface", J. Differential Geom. 13:1 (1978), 25-41. MR 520599 Zbl 0379.53016

[Wong 1977] B. Wong, "Characterization of the unit ball in $\mathbf{C}^{n}$ by its automorphism group", Invent. Math. 41:3 (1977), 253-257. MR 0492401 Zbl 0385.32016

Received February 25, 2015. Revised January 15, 2016.

JAE-CHEON JOO

DEPARTMENT OF MATHEMATICS EDUCATION

INHA UNIVERSITY

100 INHARO

NAM-GU

INCHEON 22212

SOUTH KOREA

jcjoo91@inha.ac.kr 


\title{
PACIFIC JOURNAL OF MATHEMATICS
}

Founded in 1951 by E. F. Beckenbach (1906-1982) and F. Wolf (1904-1989)

$$
\text { msp.org/pjm }
$$

\section{EDITORS}

\author{
Don Blasius (Managing Editor) \\ Department of Mathematics \\ University of California \\ Los Angeles, CA 90095-1555 \\ blasius@math.ucla.edu
}

\author{
Paul Balmer \\ Department of Mathematics \\ University of California \\ Los Angeles, CA 90095-1555 \\ balmer@math.ucla.edu \\ Robert Finn \\ Department of Mathematics \\ Stanford University \\ Stanford, CA 94305-2125 \\ finn@math.stanford.edu \\ Sorin Popa \\ Department of Mathematics \\ University of California \\ Los Angeles, CA 90095-1555 \\ popa@math.ucla.edu
}

\author{
Vyjayanthi Chari \\ Department of Mathematics \\ University of California \\ Riverside, CA 92521-0135 \\ chari@math.ucr.edu \\ Kefeng Liu \\ Department of Mathematics \\ University of California \\ Los Angeles, CA 90095-1555 \\ liu@math.ucla.edu \\ Igor Pak \\ Department of Mathematics \\ University of California \\ Los Angeles, CA 90095-1555 \\ pak.pjm@gmail.com \\ Paul Yang \\ Department of Mathematics \\ Princeton University \\ Princeton NJ 08544-1000 \\ yang@math.princeton.edu
}

\section{PRODUCTION}

Silvio Levy, Scientific Editor, production@msp.org

\section{SUPPORTING INSTITUTIONS}

ACADEMIA SINICA, TAIPEI

CALIFORNIA INST. OF TECHNOLOGY

STANFORD UNIVERSITY

UNIV. OF BRITISH COLUMBIA

UNIV. OF CALIFORNIA, BERKELEY

UNIV. OF CALIFORNIA, DAVIS

UNIV. OF CALIFORNIA, LOS ANGELES

UNIV. OF CALIFORNIA, RIVERSIDE

UNIV. OF CALIFORNIA, SAN DIEGO

UNIV. OF CALIF., SANTA BARBARA
KEIO UNIVERSITY

MATH. SCIENCES RESEARCH INSTITUTE

NEW MEXICO STATE UNIV.

OREGON STATE UNIV.
Daryl Cooper

Department of Mathematics

University of California

Santa Barbara, CA 93106-3080 cooper@math.ucsb.edu

Jiang-Hua Lu

Department of Mathematics

The University of Hong Kong

Pokfulam Rd., Hong Kong

jhlu@maths.hku.hk

$$
\text { Jie Qing }
$$

Department of Mathematics

University of California

Santa Cruz, CA 95064

qing@ cats.ucsc.edu

\author{
UNIV. OF CALIF., SANTA CRUZ \\ UNIV. OF MONTANA \\ UNIV. OF OREGON \\ UNIV. OF SOUTHERN CALIFORNIA \\ UNIV. OF UTAH \\ UNIV. OF WASHINGTON \\ WASHINGTON STATE UNIVERSITY
}

These supporting institutions contribute to the cost of publication of this Journal, but they are not owners or publishers and have no responsibility for its contents or policies.

See inside back cover or msp.org/pjm for submission instructions.

The subscription price for 2016 is US $\$ 440 /$ year for the electronic version, and \$600/year for print and electronic.

Subscriptions, requests for back issues and changes of subscriber address should be sent to Pacific Journal of Mathematics, P.O. Box 4163, Berkeley, CA 94704-0163, U.S.A. The Pacific Journal of Mathematics is indexed by Mathematical Reviews, Zentralblatt MATH, PASCAL CNRS Index, Referativnyi Zhurnal, Current Mathematical Publications and Web of Knowledge (Science Citation Index).

The Pacific Journal of Mathematics (ISSN 0030-8730) at the University of California, c/o Department of Mathematics, 798 Evans Hall \#3840, Berkeley, CA 94720-3840, is published twelve times a year. Periodical rate postage paid at Berkeley, CA 94704, and additional mailing offices. POSTMASTER: send address changes to Pacific Journal of Mathematics, P.O. Box 4163, Berkeley, CA 94704-0163.

PJM peer review and production are managed by EditFLOW ${ }^{\circledR}$ from Mathematical Sciences Publishers.

PUBLISHED BY

\section{I. mathematical sciences publishers}

nonprofit scientific publishing

http://msp.org/

(C) 2016 Mathematical Sciences Publishers 


\section{PACIFIC JOURNAL OF MATHEMATICS}

Volume $285 \quad$ No. $1 \quad$ November 2016

Iwahori-Hecke algebras for Kac-Moody groups over local fields

Nicole BARDY-PANSE, StÉPHANE GAUSSENT and GuY

ROUSSEAU

A classification of spherical conjugacy classes

MAURo Costantini

Affine weakly regular tensor triangulated categories

IVo DELL'AMBROGIO and DONALD STANLEY

Involutive automorphisms of $N_{\circ}^{\circ}$-groups of finite Morley rank

ADRIEN DELORO and ÉRIC JALIGOT

Schur-Weyl duality for Deligne categories, II: The limit case

INNA ENTOVA AIZENBUD

A generalization of the Greene-Krantz theorem for the semicontinuity 225 property of automorphism groups

JAE-CHEON JOO

Gradient estimates for a nonlinear Lichnerowicz equation under general geometric flow on complete noncompact manifolds

LIANG ZHAO and SHOUWEN FANG 